\title{
Analytical convergence in surrogate control matrices
}

\author{
Robert MacNeill*,1 \\ ${ }^{1}$ Department of Bioanalytical Services, Envigo, Mettlers Road, East Millstone, NJ 08875-2360, USA \\ *Author for correspondence: Tel.: +1 732873 2550; Fax: +1 732873 3992; robert.macneill@envigo.com
}

First draft submitted: 17 July 2018; Accepted for publication: 20 July 2018; Published online: 20 September 2018

Keywords: convergence $\bullet$ parallelism • surrogate matrix

Biomarkers continue to become increasingly important in the drug development process in recent times, and accordingly there has been more drive to find means of establishing rugged bioanalytical methods for their quantification. The most popular means of bioanalytical quantification of biomarkers using LC-MS is the so-called surrogate matrix approach. I am very pleased to write the foreword to this special themed issue which is focused on the major fundamental analytical aspect that lends reliability to methods that adopt this approach.

\section{Bringing surrogate matrices methodologically closer to authentic matrices}

With the surrogate matrix regime, calibration standards and often a portion of the quality control samples are spiked and prepared in an alternative matrix or simple solution, free of the compound of interest or for which analyte levels are known to be far below the interferent levels. Clearly, the use of surrogate matrices with an absence of significant levels of the compound of interest is pivotal and this comes with a clear caveat of great importance, the focal point of this issue. For best performance amenable ultimately to method validation, with regard to the influences on analyte and internal standard peak area responses, the chosen surrogate matrix must adequately mimic the characteristic output from the genuine matrix within the selectivity aggregate of the complete LC-MS methodology. This is the essence of parallelism.

The subject matter is peppered with very intriguing thinking points, not even to mention comparisons with the main competitor approach of surrogate analyte.

With the inherent challenges, method development times can be prohibitively long. This is the main disadvantage of this approach. The issues that lead to this are inherent in the means. Compositions of biological fluids, especially certain components or classes of components, are known to have a profound effect on peak areas in bioanalytical LC-MS methods, even with laborious sample extractions and powerful chromatography. This leads to the very real prospect of any calibration standards prepared in a composition distinct to the authentic sample matrix composition showing a biased response, giving clear implications for the accuracy of resultant calculated concentration data.

In the interests of reducing these method development times and other resource, to make the methodology only as good as it needs to be is a concept widely accepted when it comes to being able to use a method to verify significant biomarker concentration changes. A biomarker assay is not a PK assay, after all. However, would it not be ideal to avoid the prospect of skimping on quality of method performance if it was at our fingertips to do so, perhaps thanks to fresh insight and novel methodologies?

Is it a valid point of view to consider that the simpler and more interferent-free the makeup of the surrogate matrix, the better the method will perform in terms of correlation with samples in authentic matrix? To continue in this questioning vein, can solution calibration standards be considered a good default starting point for method development or is it wiser to work with as close a mimic as possible to the authentic matrix composition? Furthermore, if additives are required in solution in order to negate nonspecific adsorptive effects, should this be translated to samples in authentic matrix? How important is the material the sample container vessels are composed of, especially if nonspecific adsorption is suspected or anticipated and how will storage temperature influence this? These questions I hope give a decent taste of what is dealt with in this arena. 


\section{Recent thinking \& empirical outcomes}

The articles in this issue constitute a snapshot of the current thinking about the best angles of the surrogate matrix approach and provide a couple of examples of surrogate matrix application, which give much food for thought. The first is a white paper coming out of a recent Japan Bioanalysis Forum meeting, detailing outcomes of the discussions concerning the best selection strategy for surrogate matrices as applied to both LC-MS and ligand binding assays [1]. Following this, there is a fascinating comparison of data, obtained by Monošík and Dragsted, using dried urine swab samples against wet urine samples for the quantitative determination by UHPLC-MS of a suitably chemically diverse range of dietary metabolites [2]. Subsequently there is a report of the successful LC-MS/MS method validation by Ferreira et al., using a water-based surrogate matrix, of dexamethasone in human aqueous humor [3]. Finally, we have the very intriguing and telling piece by Incamps et al. [4] showing the possible effects of the plasma and serum matrix constituents on mass spectrometric-based measurement of protein analytes.

So, amidst the thought-provoking outcomes, there are many points for discussion and further work. The special challenges that come with endogenous compound quantification are numerous, but good chemistry, sound application and sharing of knowledge give us the well-trodden way forward.

I wish you pleasant reading.

\section{Financial \& competing interests disclosure}

The author has no relevant affiliations or financial involvement with any organization or entity with a financial interest in or financial conflict with the subject matter or materials discussed in the manuscript. This includes employment, consultancies, honoraria, stock ownership or options, expert testimony, grants or patents received or pending, or royalties.

No writing assistance was utilized in the production of this manuscript.

\section{References}

1. Wakamatsu A, Ochiai S, Suzuki E et al. Proposed selection strategy of surrogate matrix to quantify endogenous substances by Japan Bioanalysis Forum DG2015-15. Bioanalysis 10(17), 1349-1360 (2018).

2. Monošík R, Dragsted L. Dried urine swabs as a tool for monitoring metabolite excretion. Bioanalysis 10(17), 1371-1381 (2018).

3. Ferreira M, Marquez C, dos Santos D et al. Validation of direct method to quantify dexamethasone in human aqueous humor by LC-MS/MS. Bioanalysis 10(17), 1361-1370 (2018).

4. Incamps A, Saez-Boiteau C, Tiede S et al. Impact of biological matrix on inflammatory protein biomarker quantification based on targeted mass spectrometry. Bioanalysis 10(17), 1383-1399 (2018). 